\title{
The JUICE ultraviolet spectrograph: a next-generation compact UVS for ESA's JUICE mission
}

Michael Davis, G. Randall Gladstone, Kurt Retherford, Steven Persyn

Michael W. Davis, G. Randall Gladstone, Kurt D. Retherford, Steven C. Persyn, "The JUICE ultraviolet spectrograph: a next-generation compact UVS for ESA's JUICE mission," Proc. SPIE 11852, International Conference on Space Optics - ICSO 2020, 1185226 (11 June 2021); doi: $10.1117 / 12.2599357$

SPIE Event: International Conference on Space Optics - ICSO 2021, 2021, Online Only 


\section{International Conference on Space Optics-ICSO 2020}

Virtual Conference

30 March-2 April 2021

Edited by Bruno Cugny, Zoran Sodnik, and Nikos Karafolas
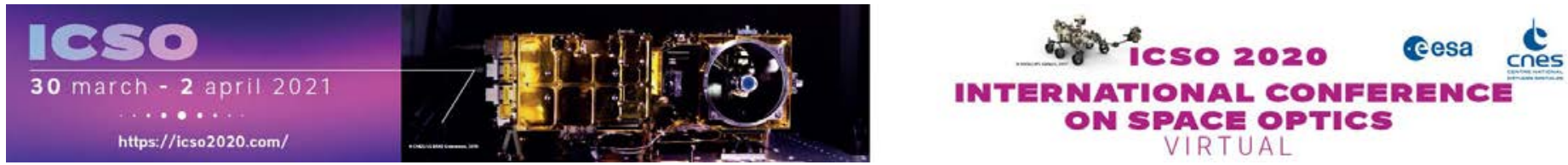

\section{The JUICE ultraviolet spectrograph: a next-generation compact UVS for ESA's JUICE mission}

\section{Cesa issopoceatings lecnes}




\title{
The JUICE ultraviolet spectrograph - a next-generation compact UVS for ESA's JUICE mission
}

\author{
Michael W. Davis ${ }^{* a}$, G. Randall Gladstone ${ }^{\mathrm{a}, \mathrm{b}}$, Kurt D. Retherford ${ }^{\mathrm{a}, \mathrm{b}}$, Steven C. Persyn ${ }^{\mathrm{a}}$ \\ ${ }^{a}$ Southwest Research Institute, 6220 Culebra Road, San Antonio, TX, USA 78238-5166; \\ bUniversity of Texas San Antonio, San Antonio, TX, USA
}

\begin{abstract}
Four compact planetary ultraviolet spectrographs have been built by Southwest Research Institute and successfully operated on different planetary missions: Rosetta-Alice, New Horizons-Alice, LRO-LAMP, and Juno-UVS. A fifth, JUICE-UVS, has been delivered to Airbus in preparation for ESA's JUICE mission to Ganymede and the Jovian system. This spectrograph features advancements that improve spatial resolution, maximum instantaneous count rates, and radiation background rejection when compared to previous spectrographs. A high-resolution port (HP) is added to improve the spatial resolution of the base UVS design by a factor of approximately 4, at a cost of 16 times worse signal-to-noise. The new design also allows solar occultations at Jupiter and its moons by reworking the SOCC that flew on New HorizonsAlice. JUICE-UVS will explore the Galilean satellites, investigate the dynamics and energetics of Io's atmosphere and torus, and characterize the dynamics and vertical structure of Jupiter's upper atmosphere.
\end{abstract}

Keywords: UV spectroscopy, JUICE

\section{INTRODUCTION}

Southwest Research Institute (SwRI) was chosen by ESA and NASA to build the ultraviolet spectrograph for ESA's JUICE mission in February 2013, hereafter referred to as JUICE-UVS. This spectrograph is the fifth in a series of six UV spectrographs delivered or under development by SwRI for various NASA and ESA missions. The first, Rosetta-Alice ${ }^{1}$, launched in March 2004, reached comet 67P/Churyumov-Gerasimenko in November 2014, and ended its mission by planned impact with the comet in September 2016. The second, PERSI-Alice (or P-Alice) ${ }^{2}$ was launched on New Horizons in January 2006, encountered the Pluto-Charon system in July 2015, and performed the first flyby reconnaissance of a Kuiper Belt object (Arrokoth) in January 2019. The Lyman-Alpha Mapping Project (LAMP) was a modification of the $P$ Alice instrument built for the Lunar Reconnaissance Orbiter (LRO) ${ }^{3}$. This instrument successfully launched in June 2009, completed its initial one-year survey of the lunar surface in September 2010, and is currently in its eleventh year of extended science operations. Juno-UVS $S^{4}$ launched in August 2011 aboard the Juno mission, reached Jupiter in July 2016, and as of early 2021 is still in its prime science phase. Europa-UVS is under development for the Europa Clipper mission and is scheduled to launch in 2024.

JUICE-UVS was completed by SwRI and delivered to Airbus Defence Systems in Friedrichshafen, Germany, for integration to the JUICE spacecraft in February 2020. JUICE-UVS builds heavily upon the heritage of the previous SwRI UV spectrographs while incorporating modifications tailored for the JUICE mission. First, JUICE-UVS includes tantalum shielding like Juno-UVS to protect itself from the harsh Jovian radiation environment and reduce detector background rates. Second, a modification of the solar occultation channel from P-Alice is included to allow solar occultation observations at Jupiter and its moons, where the Sun is approximately 30 times brighter than at Pluto. Third, a second aperture door is included to act as a high-resolution "aperture stop" and allow observations with greater spatial resolution than previous UVS instruments ${ }^{6}$. Finally, the detector has been upgraded to include an atomic layer deposition (ALD) coating to minimize sensitivity loss induced by accumulated lifetime fluence ${ }^{7}$.

*mdavis@swri.edu 


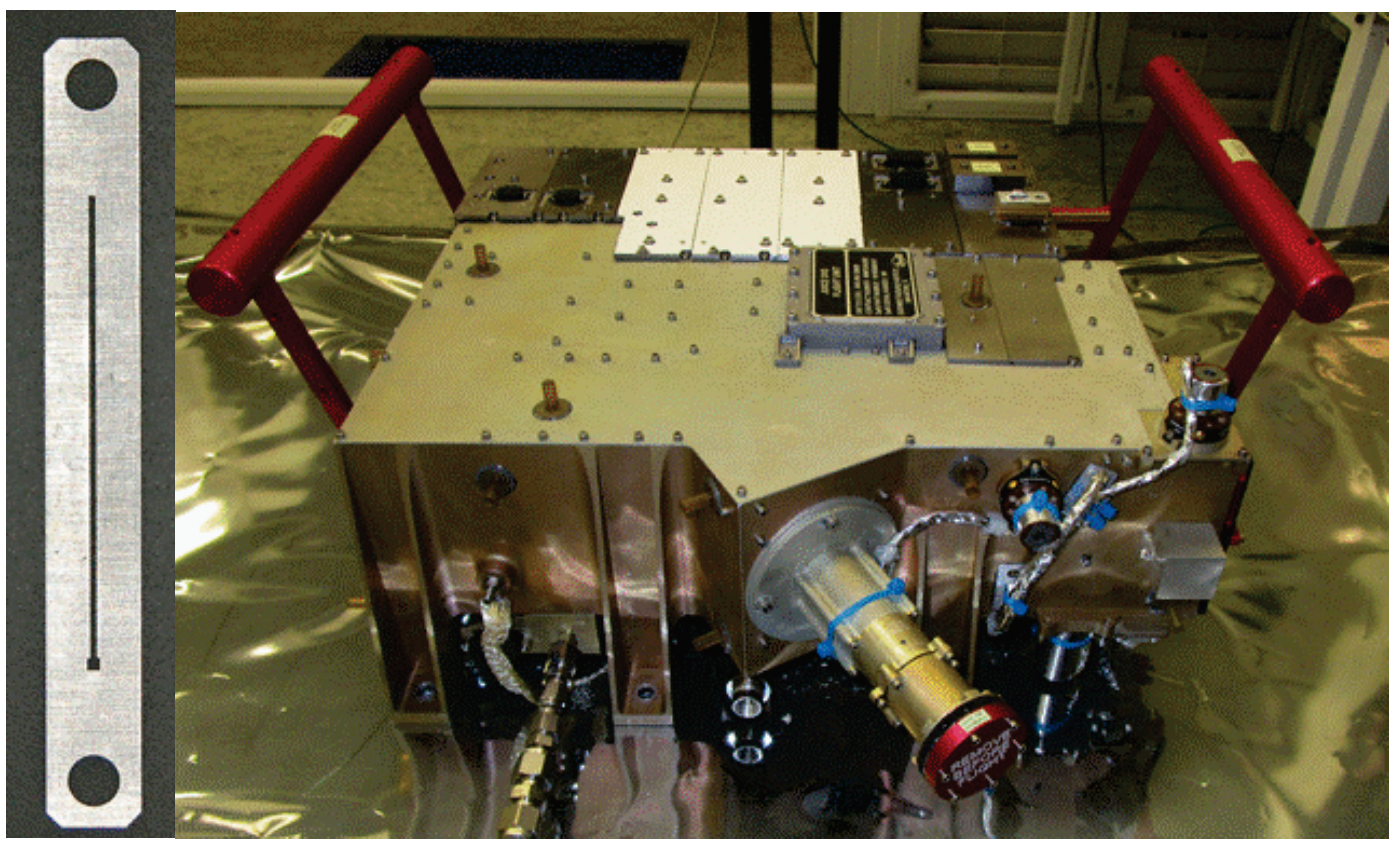

Figure 1. (left) The JUICE-UVS slit, consisting of a long thin section $\left(7.3^{\circ} \times 0.1^{\circ}\right)$ and a small box $\left(0.2^{\circ} \mathrm{x} 0.2^{\circ}\right)$ for solar input. (right) The completed JUICE-UVS instrument shortly before delivery to ESA.

\section{INSTRUMENT DESCRIPTION}

\subsection{Overview}

Light enters the telescope section via one of three apertures: a $40 \times 40 \mathrm{~mm}$ square airglow port (AP, the main aperture), a $10 \times 10 \mathrm{~mm}$ square high resolution (HP) port, or a $0.25 \mathrm{~mm}$ diameter pinhole in the solar port (SP). Light that enters the $\mathrm{SP}$ is then reflected off a small pickoff mirror. In all three cases, the entering light is then collected and focused by an offaxis paraboloidal (OAP) primary mirror $(120 \mathrm{~mm}$ focal length) onto the spectrograph entrance slit. The OAP and grating are coated with aluminum with a magnesium fluoride overcoat $\left(\mathrm{Al} / \mathrm{MgF}_{2}\right)$ to maximize reflection at wavelengths $>115$ $\mathrm{nm}$. The SP pickoff mirror is coated with gold, which is approximately $35 \%$ reflective in the UV. This reduced throughput enables solar observations at $5 \mathrm{AU}$. The entrance slit, as shown in Figure 1, has a FOV of $0.1^{\circ}$ in the dispersion direction and $7.3^{\circ}$ in the spatial direction (i.e. perpendicular to the dispersion direction). The slit contains an additional small $0.2^{\circ}$ box at one end to collect sunlight from the SP. The completed flight JUICE-UVS is also shown in Figure 1.

Light passing through the entrance slit falls onto a toroidal holographic grating with 1600 grooves $/ \mathrm{mm}$ and a radius-ofcurvature of $150 \mathrm{~mm}$ in the dispersion plane. The light is then dispersed onto a microchannel plate (MCP) detector with a cross-delay line (XDL) readout scheme ${ }^{7}$. The 2-D (4096 x 4096)-pixel format, MCP detector uses a solar-blind cesium iodide (CsI) photocathode that covers the range 50-204 $\mathrm{nm}$.

The detector's MCP configuration is a Z-stack that is cylindrically curved to match the $150-\mathrm{mm}$ Rowland circle diameter to optimize spectral and spatial focus across the JUICE-UVS passband ${ }^{7}$. The detector electronics provide two stimulation pixels that can be turned on to check data throughput and acquisition modes without the need to apply high voltage to the MCP stack. The MCP pulse-height information is output as 8 bits in the 4-byte output for every photon.

The JUICE-UVS housing is a monolithic piece of aluminum to minimize thermal defocus between the aluminum optics and grating. Many small overlapping tantalum plates were mounted to the aluminum housing to shield against the $\mathrm{MeV}$ level electrons present during prime Jovian operations. All optical surfaces mount to aluminum. Furthermore, the spacing between tantalum plates allows for thermal expansion of the aluminum without deforming the tantalum plates or compromising the effectiveness of the radiation shielding. A similar design was successfully implemented on Juno-UVS .

JUICE-UVS is controlled by a field-programmable gate array (FPGA) with an instantiated 8051 micro-controller, and utilizes lightweight, compact, surface mount electronics to support the detector as well as instrument interface electronics. 
The resulting design enjoys strong parts-level heritage from previous builds, especially Juno-UVS $S^{4}$ A summary of the performance of JUICE-UVS as measured during the calibration tests is given in Table 1.

Table 1. JUICE-UVS Measured Performance Summary

\begin{tabular}{|l|l|}
\hline \hline Bandpass: & $50-204 \mathrm{~nm}$ \\
\hline \multirow{2}{*}{ Spectral Resolution: } & $\sim 0.5-0.8 \mathrm{~nm}$ (center of slit; wavelength dependent) \\
\cline { 2 - 2 } & $\sim 1.5 \mathrm{~nm}$ (near edge of slit) \\
\hline Spatial Resolution: & $0.07^{\circ}$ (near $90 \mathrm{~nm}$ at center of slit) \\
\hline & $0.29^{\circ}($ near edge of slit) \\
\hline Pixel Plate Scale & $0.0092^{\circ} \pm 0.0001^{\circ}$ (spatial); $\sim 0.106 \mathrm{~nm}$ (spectral) \\
\hline Peak Effective Area: & $0.8 \mathrm{~cm}^{2}(1216 \AA)$ \\
\hline Mass/Power & $19.4 \mathrm{~kg} / 7.5 \mathrm{~W}$ \\
\hline
\end{tabular}

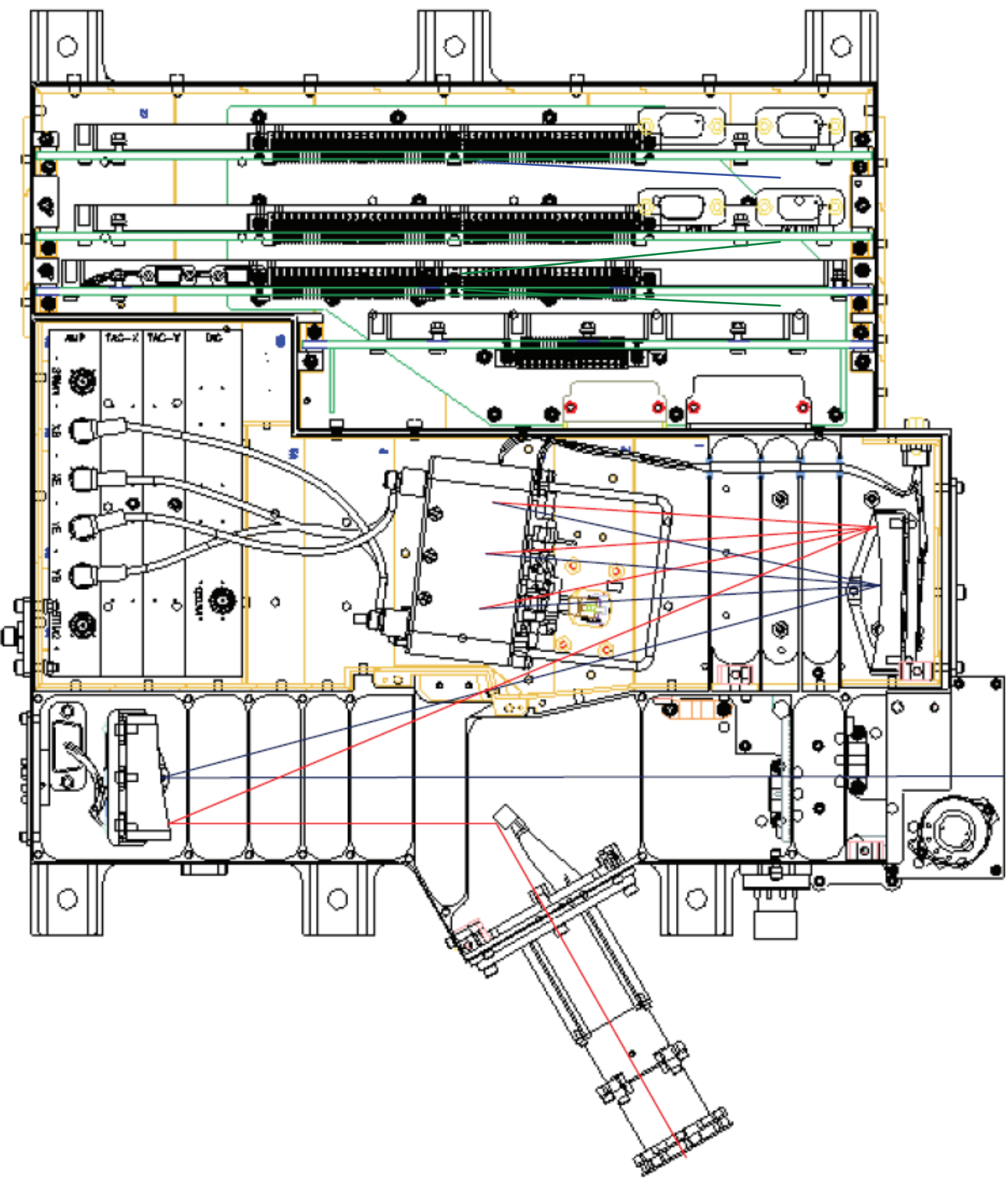

Figure 2. An opto-mechanical schematic of JUICE-UVS showing light rays traced through the main aperture door (blue) and the solar port (red), passing through a focus at the slit, diffracted off the grating, and imaged onto the detector. 


\subsection{Opto-Mechanical Design Overview}

The housing and optics are all made from aluminum, resulting in an athermal design. The OAP mirror, solar port mirror, and diffraction grating are machined from monolithic pieces of aluminum (including mounting features) and polished to minimize surface roughness and scatter. The OAP mirror and solar port mirror are coated with electroless nickel before polish to prevent oxidation. All three optical elements are given a post-polish coating of $\mathrm{Al} / \mathrm{MgF}_{2}$ to optimize FUV reflectivity. The housing features multiple baffle vanes in both the telescope and spectrograph section to minimize stray light. Zero-order light from the grating reflects off a shiny surface on the detector housing; the reflected light is then trapped within a zero-order light trap attached to the spectrograph inner wall. The solar port aperture is canted $60^{\circ}$ away from the instrument boresight to maintain solar keepout zones as well as avoid interference with the spacecraft solar panels. The tantalum shielding consists of a number of interleaving plates that allow for thermal mismatch between the aluminum housing and the plates while maintaining contiguous $4 \pi$ steradian coverage. A labeled opto-mechanical schematic of JUICE-UVS is shown in Figure 2, including light rays representing both AP and SP input.

\subsection{Detector and Detector Electronics}

The JUICE-UVS detector and electronics are a photon-counting UV detector system based on decades of flight heritage, and four previous UVS detector designs. The detector MCP stack acts as a photomultiplier; a CsI photocathode has been applied to the top plate to improve quantum efficiency at FUV wavelengths. A photon that strikes the photocathodecoated front plate is transformed into a charge that is then multiplied by approximately $6 \times 10^{6}(\sim 1 \mathrm{pC})$ as the charge travels through the MCP pores. The charge cloud exits the MCP stack and strikes the cross-delay line anode. The anode contains two serpentine wires that span the detective area in perpendicular directions (X-and Y-directions). The charge propagates through the wires such that the "start" and "stop" times are recorded by the detector electronics in each direction. These "start" and "stop" times are then processed into X- and Y-positions and sent to the main instrument electronics. The process starts again with each UV photon that strikes the detector, resulting in a true photon-counting detection system.

The detector consists of three curved microchannel plates in a z-stack read out by a cross-delay line anode. The curvature of these plates $(7.5 \mathrm{~cm}$ radius) matches the UVS Rowland circle radius $(15 \mathrm{~cm})$. A repeller grid above the MCP stack reflects stray photoelectrons, increasing detective quantum efficiency. The detector assembly resides within a vacuum enclosure containing a one-time-in-flight opening door. The door may be manually reset and latched during ground testing. The door also holds a fused silica window to allow ground testing at longer FUV wavelengths while minimizing susceptibility to radiation-induced fluorescence in flight. The detector vacuum housing may be independently pumped via a port on the housing bottom. A polished nickel strip is attached to the housing on one side of the door and acts as a zeroorder reflector. Four SMA connectors on the detector backside read out the anode, and two HV connectors provide the high voltage and return.

The detector electronics process the anode output into usable image information. The detector output is digitized into a $4096 \times 4096$ array of pixels, out of which the active area encompasses 790 pixels in the spatial direction and 1487 pixels in the spectral direction. This digital range is an increase over the 2048 (spectral) x 256 (spatial) resolution of the JunoUVS detector electronics. The detector electronics can also provide two "stimulation" pixels outside of the active area that may be used to test the instrument data processing in the absence of high voltage. The positions of these "stim" pixels may also be used to correct detector performance for variations due to temperature. Furthermore, pulse height information is recorded for every event with up to 8-bit resolution, an increase from the 5-bit resolution featured on Juno-UVS. If the full detector resolution is utilized, the pulse height and position information result in a 4-byte data word for every event.

The data words are processed by the instrument command and data handling (C\&DH) electronics in one of two fashions. The first, called "pixel list mode," assembles a list of event positions and pulse heights, interleaved with a "time hack" that records the event time every 0.001 to 0.25 seconds. The resolution of the event positions, pulse heights, and time hack are programmable so that science return can be maximized while minimizing recorded data volume. The second mode is "histogram" mode, which assembles a "traditional" image with a maximum area of $1 \mathrm{MB}$ and maximum "well depth" of 16 bits. While the full $4 \mathrm{k} \times 4 \mathrm{k}$ detector space cannot be sampled in histogram mode due to the $1 \mathrm{MB}$ size restriction, the image can be sampled at $2 \mathrm{k} \times 512$ or $512 \times 2 \mathrm{k}$ to maximize spectral or spatial resolution, respectively. Furthermore, the sampling can be further reduced in either direction to condense data volume depending on the needed spatial or spectral resolution. A lookup table (LUT) in software controls the resolution of the stored histogram so that any image size is possible as long as it does not exceed $1 \mathrm{MB}$. This flexibility in data sampling allows maximum science return on all observations while minimizing data volume and downlink needs. 
The detector electronics feature two more improvements over the XDL system flown on Juno-UVS. First, the speed of the system has been improved so that it records a $10 \%$ deadtime loss at $130 \mathrm{kHz}$ input rates instead of the $85 \mathrm{kHz}$ of JunoUVS. This improved deadtime allows for more data events to be recorded in the high background radiation environment near the Galilean moons. The second improvement is the addition of a dedicated analog-to-digital converter (ADC) to the pulse height electronics. The Juno-UVS electronics suffered from a drop in recorded pulse height position as count rate increased, making the absolute recorded pulse height level unreliable at $>100 \mathrm{kHz}$ input rates. JUICE-UVS will not suffer from this problem due to the additional ADC, making the pulse height a more useful tool in discriminating between photon (science) and radiation (noise) events.

\subsection{JUICE-UVS Electrical Design}

JUICE-UVS features dual-string high- and low-voltage power supplies (HVPS and LVPS, respectively), redundant actuators, and a single-string C\&DH. The LVPS receives $28 \mathrm{~V}$ from the spacecraft and passes it along to the instrument heaters and actuators. It also converts the $28 \mathrm{~V}$ power to the $\pm 6.12 \mathrm{~V}$ and $+3.3 \mathrm{~V}$ power needed by the other instrument electronics. The HVPS is capable of generating up to $-5400 \mathrm{~V}$ for detector operation, although nominal operational voltage is $-4.45 \mathrm{kV}$ at room temperature. A block diagram giving further details about the JUICE-UVS electronics is shown in Figure 3.

\section{CALIBRATION}

At the completion of instrument integration activities, JUICE-UVS underwent an environmental test program that included vibration, electromagnetic compatibility, and thermal-vacuum testing. Following these tests, radiometric characterization and absolute calibration of the instrument were performed in our UV calibration facility located in the SwRI Space Science and Engineering Division ${ }^{8}$. The radiometric characterization tests included the following: detector dark count rate, wavelength calibration, and spectral and spatial point source function (PSF) vs. wavelength. Finally, the absolute effective area was measured as a function of wavelength through both the AP and SP channels. The results are summarized in the following pages; further details may be found in the JUICE-UVS calibration summary paper.

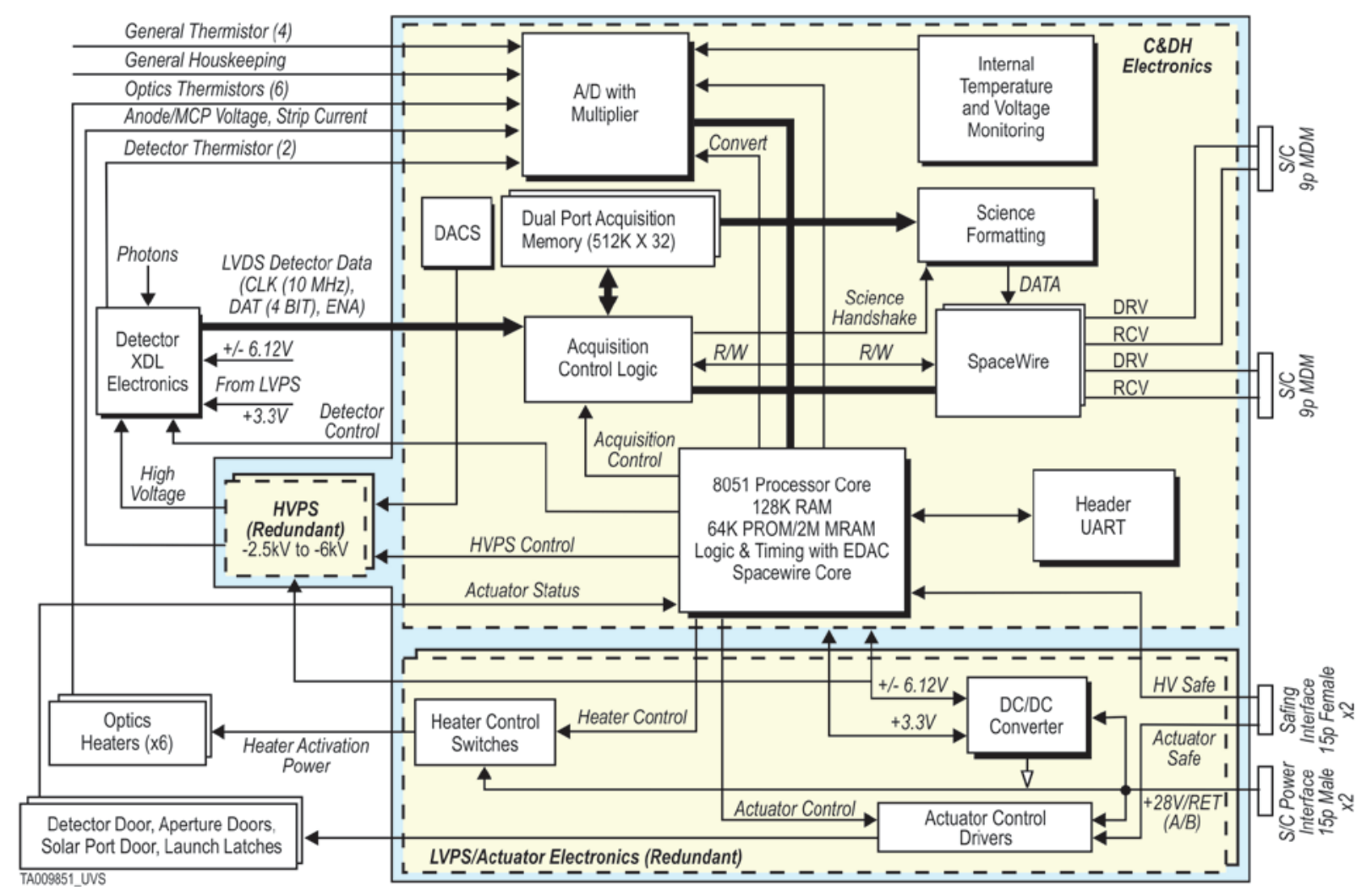

Figure 3. JUICE-UVS Electrical Block Diagram. 


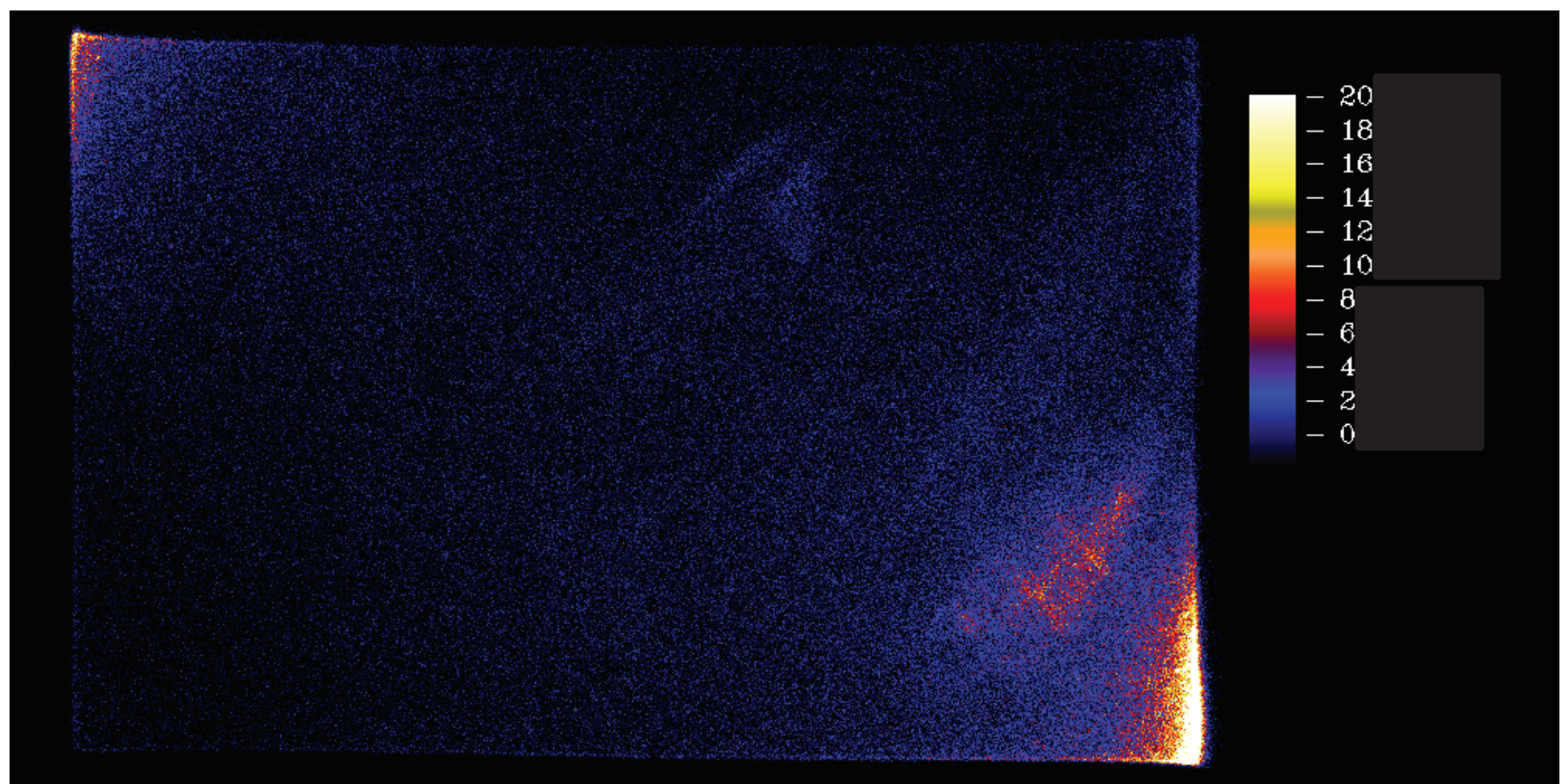

Figure 4. Image histogram of the dark pixel list exposures for the entire 14 hours of accumulated exposure. The color bar represents total counts in the accumulated exposure.

\subsection{Dark Count Rate}

A series of long dark observations were taken over multiple days and times throughout the calibration process. These observations have been co-added to produce a 14-hour dark frame. The dark measurements were taken with the detector operating at $-4450 \mathrm{~V}$ (the nominal $\mathrm{HV}$ level for room temperature at delivery) and all chamber windows covered with foil to minimize any stray light in the calibration chamber. Figure 4 shows an image of the combined 14 hours of dark exposure. There are "warm" spots at the upper left and at the bottom right corner of the active area that correspond to the standalone detector performance previously measured ${ }^{7}$. The overall dark rate has decreased in the year between standalone detector testing and instrument calibration, indicating that further exposure to vacuum will continue to reduce the dark rate. The maximum accumulated counts in a single pixel were 2056 counts; the average pixel has approximately 1 count accumulated over the cumulative 14-hour exposure.

\subsection{Point Spread Functions}

The spectral and spatial point spread functions (PSFs) were measured at $0.5^{\circ}$ intervals along the length of the slit using argon and neon gas in the hollow-cathode UV light source. Images were acquired beginning at the center of the slit (spatial offset of $0^{\circ}$ ) and at offset angles of $\pm 0.5^{\circ}, \pm 1.0^{\circ}, \pm 1.5^{\circ}, \pm 2.0^{\circ}, \pm 2.5^{\circ}, \pm 3.0^{\circ}$, and $\pm 3.5^{\circ}$ with respect to slit center. The test monochromator was set at zero order, the input slit was set to $10 \mu \mathrm{m}$ to avoid local gain depression on the detector, and the output slit width was set to $50 \mu \mathrm{m}$. The stim pixels were turned on for each 300 -second exposure. The raw spectral images and the spectral pixel solution were combined to determine the spectral resolution as a function of spatial position and wavelength. For each exposure, the image row with the maximum number of counts was used to fit a series of Gaussians for each identified emission line in the spectral axis. There was no distortion or tilt correction on the spectral lines; instead, each spectral observation was collapsed into a single row and fit with a 1-dimensional Gaussian. All the measured spectral PSFs across the JUICE-UVS passband meet the specification of $<2 \mathrm{~nm}$ FWHM, and exceed the goal of $<1.2 \mathrm{~nm}$ FWHM for all but a few wavelengths and spatial positions. Figure 5 shows the FWHM of the spectral PSF as a function of y-position at a number of wavelengths of interest across the passband.

The initial step of the spatial resolution analysis collapsed the separate argon and neon measurements at each y-position along the $\mathrm{x}$-direction to measure the Gaussian width in the $\mathrm{y}$-direction. The images were broken up into discrete positions separated by 20 pixels in the $\mathrm{x}$-direction, with each unit summed over a bin 30 pixels wide. For each y-position (approximately equivalent to $0.5^{\circ}$ in angular space), these bins were fit by a Gaussian to determine the spatial resolution in pixels. This width was converted to angular space assuming a $0.0092 \%$ pixel conversion. This conversion factor is based on both the $0.5^{\circ}$ steps of the motion table and previous measurements with a collimated light source attached to a 
goniometer. The PSF meets the requirement of $<0.3^{\circ}$ for all wavelengths, with a maximum measured PSF of $0.28^{\circ}$ at long wavelengths. Furthermore, the smallest measured PSF is at $93 \mathrm{~nm}$ (approximately at x-position 1200), consistent with the $90 \mathrm{~nm}$ astigmatic point of the UVS optical design.

\subsection{Wavelength Calibration}

The images taken to determine the PSF values were also used to determine the wavelength calibration. Ten different lines were identified in the Ar spectra and 12 lines in the Ne spectra. The observations for the two gases were corrected by offsetting the Ne spectra so that the residual Lyman-alpha $(121 \mathrm{~nm})$ emission seen in both $\mathrm{Ar}$ and Ne overlapped at pixel 1475. The first attempt at a wavelength solution utilized a linear fit, but this solution was rejected due to an "s" shape in the residuals. The next attempt utilized a cubic fit, which produced randomly distributed residuals between the error bars. Figure 6 shows the solution at $y=1600$. The cubic fit is such that the pixel scale varies from $0.11 \mathrm{~nm}$ at $121 \mathrm{~nm}$ to 0.095 $\mathrm{nm}$ at the short and long wavelength boundaries. The linear approximation of this cubic fit is $0.1064 \mathrm{x}-35.29$, or approximately $0.106 \mathrm{~nm} / \mathrm{spectral}$ pixel.

With the measured plate scale and offset values, the total wavelength passband across the detector active area can be computed. The active area starts at spectral pixel 777 and ends at pixel 2263. Allowing for a 5-pixel buffer in each direction to account for count pile-up and other detector edge effects, the remaining area corresponds to a total wavelength passband of 50-204 $\mathrm{nm}$. This passband satisfies the instrument specification for a minimal passband of 55-180 $\mathrm{nm}$ with at least $5 \mathrm{~nm}$ margin on each end.

\subsection{Spatial Plate Scale}

The spatial plate scale was measured by tilting JUICE-UVS on the calibration chamber scan platform over a $7.3^{\circ}$ spatial range, then illuminating the entrance aperture at $0.5^{\circ}$ steps. The total length of the illuminated $7.3^{\circ}$ length of slit section comprises $\sim 790 \pm 0.5$ pixels, which gives a per pixel plate scale of $0.0092^{\circ} \pm 0.0001^{\circ}$. This plate scale meets the instrument specification of $<0.02^{\circ}$ per pixel. This plate scale was confirmed prior to vacuum-level calibration by benchtop alignment tests with a boresight-aligned collimator attached to a goniometer. The goniometer tilted the collimator over a $7^{\circ}$ range, with a spread of $\sim 760 \pm 0.5$ pixels on the detector, confirming the plate scale of $0.0092^{\circ}$.
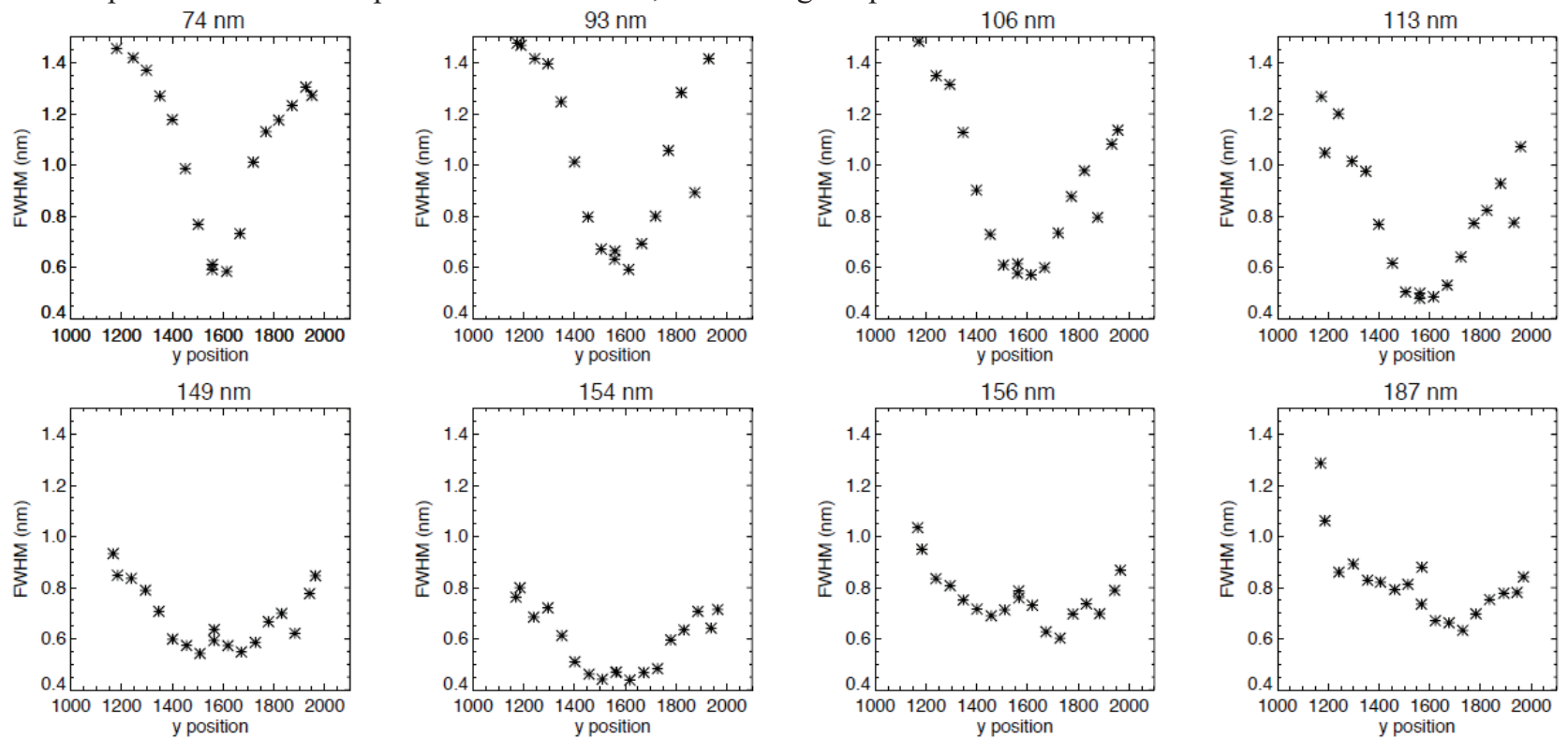

Figure 5. The measured spectral PSF at various test wavelengths as a function of row number. The PSF is under the requirement of $2 \mathrm{~nm}$ at all rows and wavelengths, and meets the goal of under $1.2 \mathrm{~nm}$ at all but the edges of the field of view at some wavelengths. 


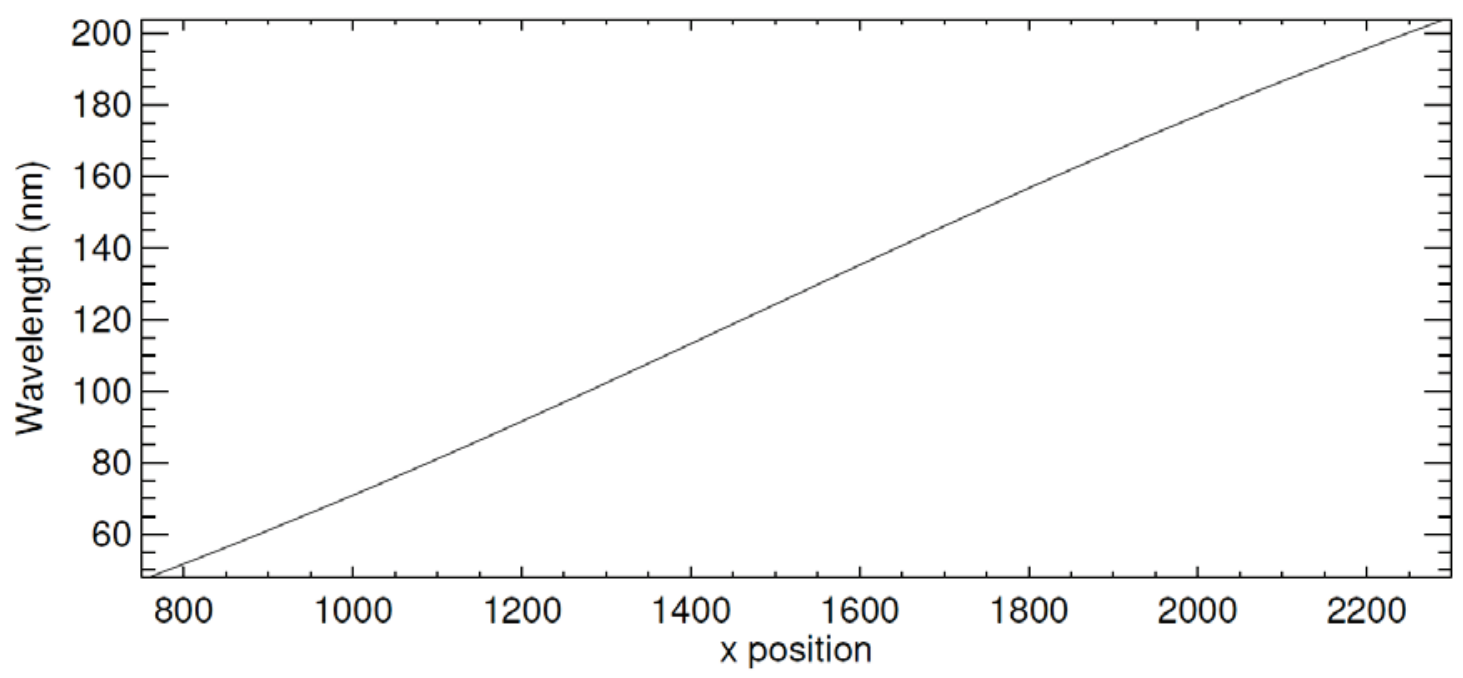

Figure 6. JUICE-UVS wavelength solution at $\mathrm{y}=1600$.

\subsection{Effective Area}

The effective area was measured across the JUICE-UVS passband at discrete wavelengths using emission lines from neon, argon, and a hydrogen/helium (5\%/95\%) gas mix covering a wavelength range of 58.4-191.6 nm. Exposures were taken with the chamber's monochromator set to the specific wavelength of the measurement. The output slit was set to provide a detector output count rate that did not show any saturation effects in the pulse-height distribution (i.e., too high a local flux on the MCP stack will cause a local gain drop that is evident in the pulse height data). Background exposures were also taken for each illuminated exposure to allow for dark subtraction.

The beam flux was quantified for each effective area measurement by illuminating a silicon ( $\mathrm{Si}$ ) photodiode (NIST diode \#06-225). In most cases, the beam flux had to be increased in intensity beyond the saturation limit of the AP channel to allow enough flux for a measurement with each photodiode. The UVS input flux was limited by inserting an aluminum plate with a precision drilled pinhole at the entrance aperture. Most effective area measurements were taken with a 2-mm diameter pinhole, aside from a few measurements near Lyman-alpha that required a $400-\mu \mathrm{m}$ diameter pinhole to prevent local saturation.

Figure 7 shows the measured effective area through the $\mathrm{AP}$ at wavelength $\lambda, A_{\text {eff }}(\lambda)$, using the following equation:

$$
A_{e f f}(\lambda)=\frac{A_{g} A_{d} R(\lambda)}{A_{p} \Phi(\lambda)},
$$

where $A_{g}$ is the geometric area of the JUICE-UVS entrance aperture $\left(16 \mathrm{~cm}^{2}\right), A_{d}$ is the geometric area of the Si photodiode $\left(1 \mathrm{~cm}^{2}\right), A_{p}$ is the geometric area of the GSE pinhole $\left(0.031 \mathrm{~cm}^{2}\right.$ or $\left.0.0013 \mathrm{~cm}^{2}\right), R(\lambda)$ is the detector count rate (corrected for the detector deadtime of $0.9 \mu \mathrm{sec}$ and subtracting the detector background), and $\Phi(\lambda)$ is the measured flux using the NIST Si photodiode. Note that the effective area requirement, as specified in the instrument specification, was met with plenty of margin. The "desired" effective area levels at wavelengths other than $125 \mathrm{~nm}$ were also met with plenty of margin.

These calculations assume the input beam is of uniform intensity over the $1 \mathrm{~cm}^{2}$ active area of the calibration diode, so that any input difference between the diode measurement and the pinhole measurement is due only to differences in collecting area. Previous measurements of the input beam show that the intensity is uniform to a few percent over a $4 \mathrm{~cm}$ $\times 1 \mathrm{~cm}$ area ${ }^{8}$, validating the calculation assumption.

The effective area was also measured through the SP at a few wavelengths where the input was bright enough to get reasonable signal-to-noise without excessive exposure time. The results of these measurements are shown in Figure 8. The maximum effective area through the SP is approximately $10^{4}$ times lower than the maximum effective area through the AP, as expected due to the smaller entrance aperture and the extra reflection off a gold-coated pickoff mirror. 


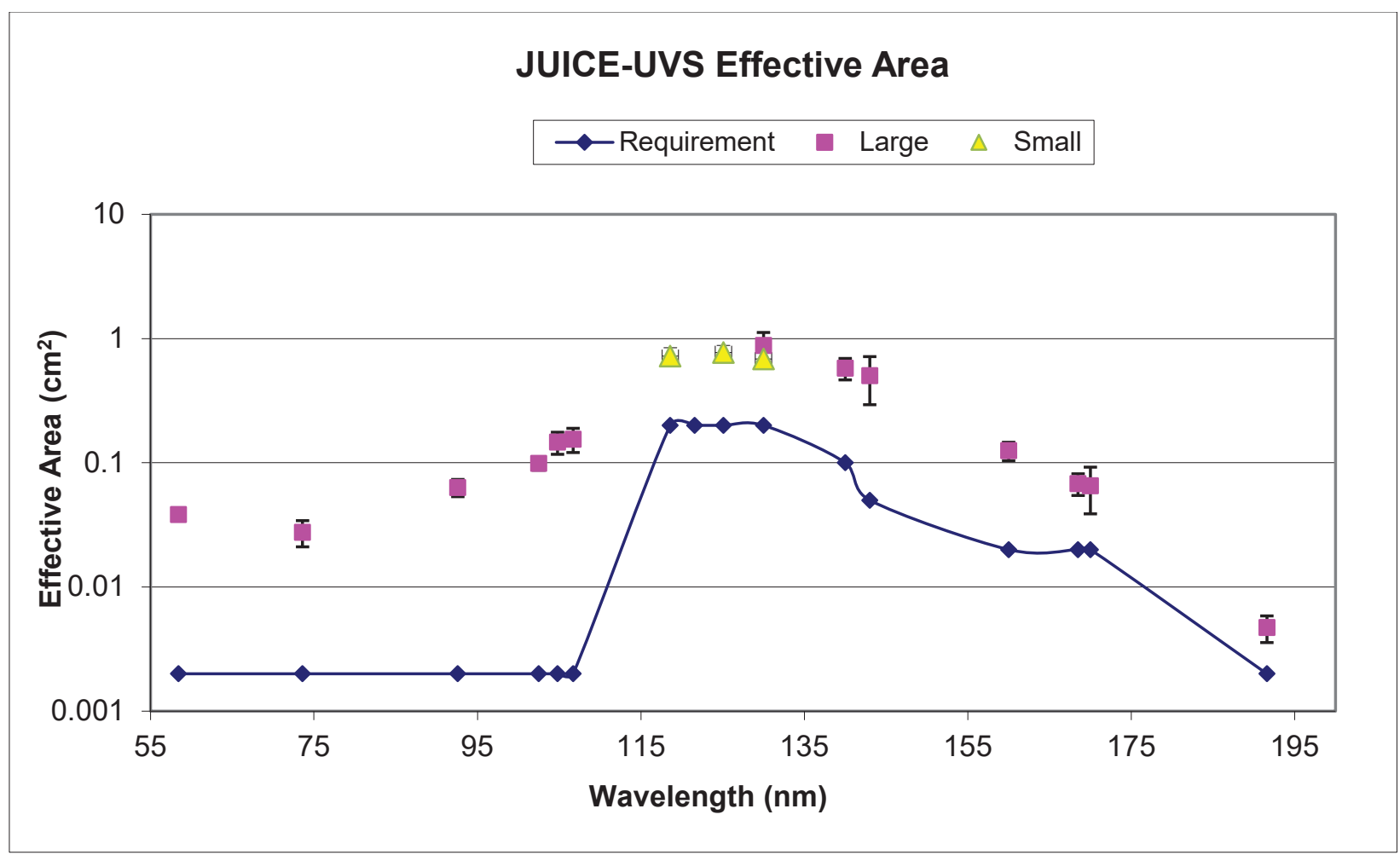

Figure 7. The measured effective area for the airglow channel (magenta squares and yellow triangles representing measurements through the large and small pinhole, respectively) plotted versus wavelength. The instrument specification effective area goal is also shown (blue diamonds).

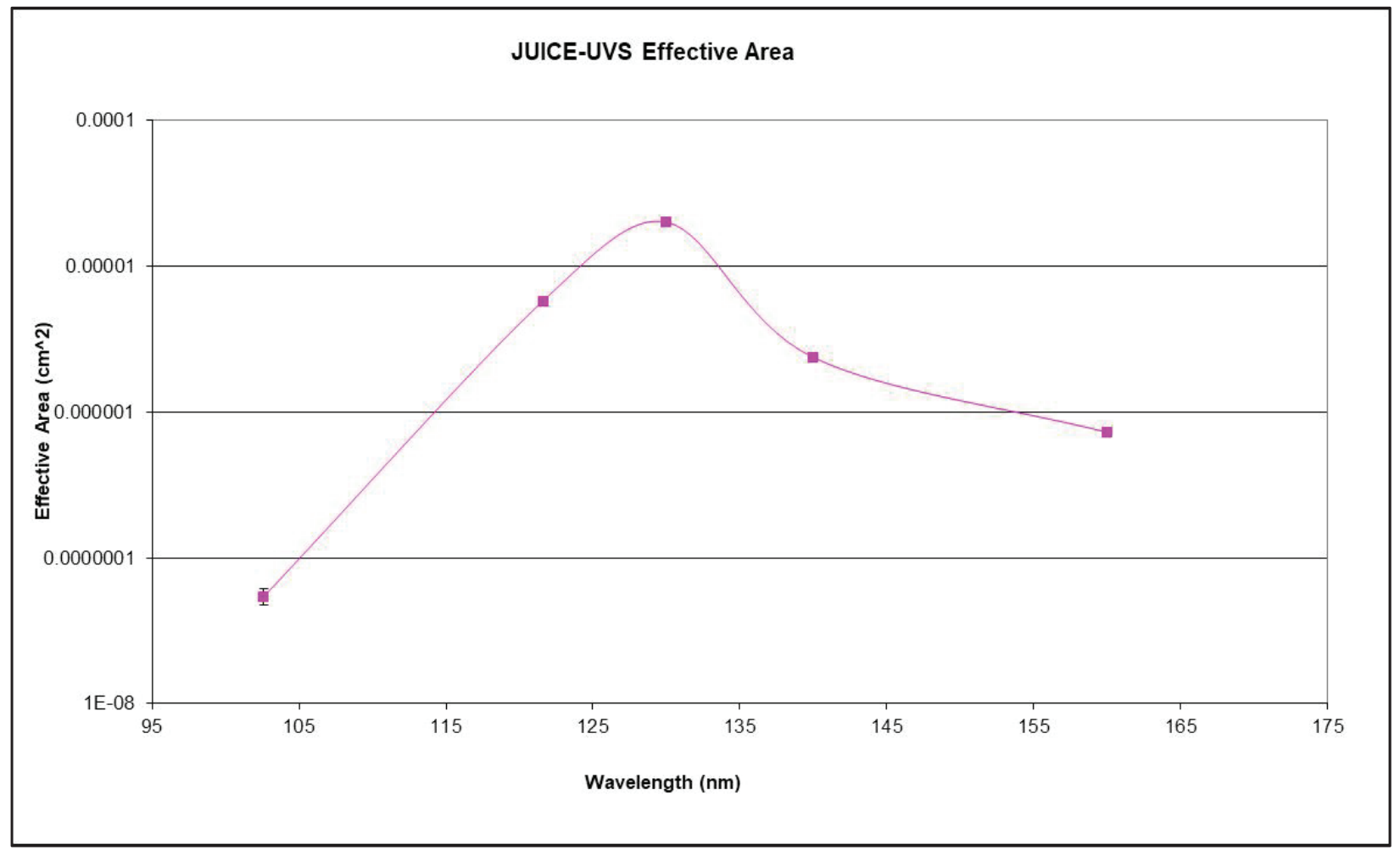

Figure 8 . The measured effective area for the solar port as a function of wavelength. 


\section{CONCLUSIONS}

JUICE-UVS has successfully completed all environmental (EMI, vibration, and thermal vacuum) and radiometric testing. UVS was delivered to Airbus for integration with the JUICE spacecraft in February 2020, and was mechanically integrated to the spacecraft in December 2020. The instrument showed no signs of performance degradation after delivery to the spacecraft, and performance will be monitored throughout the spacecraft assembly, integration, and test program. JUICEUVS met or exceeded all performance requirements, and therefore will meet the science objectives proposed for the JUICE mission. Launch is currently scheduled for 2022. An extensive in-flight calibration is planned during interplanetary cruise to verify performance, with follow-on observations of bright UV stars and the Sun planned at strategic points during the prime mission.

\section{ACKNOWLEDGEMENTS}

We wish to thank the members of the JUICE-UVS integration and test team for their support, especially Sue Baldor, Rohini Giles, Roy Graham, Thomas Greathouse, Philippa Molyneux, Kristian Persson, Norman Pelletier, Maarten Versteeg, and Brandon Walther. We wish to thank Oswald Siegmund, Adrian Martin, and John Vallerga of Sensor Sciences, LLC, for their support and provision of the UVS detector system. This work was supported by NASA contract NNM13AA38C.

\section{REFERENCES}

[1] Stern, S.A., Slater, D.C., Scherrer, J., Stone, J., Versteeg, M., A'Hearn, M.F., Bertaux, J.L., Feldman, P.D., Festou, M.C., Parker, J.Wm., and Siegmund, O.H.W., "Alice: The Rosetta Ultraviolet Imaging Spectrograph." Space Science Reviews 128, pp. 507-527 (2007).

[2] Stern, S.A., D.C. Slater, J.R. Scherrer, J.M. Stone, G.J. Dirks, M.H. Versteeg, M.W. Davis, G.R. Gladstone, J.Wm. Parker, L.A. Young, O.H.W. Siegmund "Alice: The ultraviolet imaging spectrograph aboard the New Horizons PlutoKuiper Belt mission." Space Science Reviews 140, 155-187 (2008).

[3] Gladstone, G. R., Stern, S. A., Retherford, K. D., Black, R. K., Slater, D. C., Davis, M. W.., Versteeg, M. H., Persson, K. B., Parker, J. W., Kaufmann, D. E., Egan, A. F., Greathouse, T. K., Feldman, P. D., Hurley, D., Pryor, W. R., and Hendrix, A. R., "LAMP: the Lyman Alpha Mapping Project on NASA's Lunar Reconnaissance Orbiter Mission," Space Science Reviews 150, pp. 161-181 (2010).

[4] Gladstone, G.R., Persyn, S.C., Eterno, J.S., Walther, B.C., Slater, D.C., Davis, M.W., Versteeg, M.H., Persson, K.B., Young, M.K., Dirks, G.J., Sawka, A.O., Tumlinson, J., Sykes, H., Beshears, J., Rhoad, C.L., Cravens, J.P., Winters, G.S., Klar, R.A., Lockhart, W. Piepgrass, B.M., Greathouse, T.K., Trantham, B.J., Wilcox, P.M., Jackson, M.W., Siegmund, O.H.W., Vallerga, J.V., Raffanti, R., Martin, A., Gerard, J.-C., Grodent, D.C., Bonfond, B., Marquet, B., and Denis, F., "The Ultraviolet Spectrograph on NASA's Juno Mission," Space Science Reviews 213, 447. https://doi.org/10.1007/s11214-014-0040-z (2017).

[5] Retherford, K., et al., "NASA's Europa Clipper Mission Ultraviolet Spectrograph (Europa-UVS)," 42 ${ }^{\text {nd }}$ COSPAR Scientific Assembly, Abstract id. B5.3-54-18 (2018).

[6] Davis, M.W., Osterman, S.N., Gladstone, G.R., Retherford, K.D., "High-resolution aperture shape analysis for the JUICE and Europa ultraviolet spectrographs," Proc. SPIE 11118, UV, X-Ray, and Gamma-Ray Space Instrumentation for Astronomy XXI, 1111819 (9 September 2019); https://doi.org/10.1117/12.2529668

[7] Davis, M.W., Siegmund, O.H.W., Gladstone, G.R., Martin, A., Molyneux, P.M., Retherford, K.D., Veach, T.J., Vallerga, J.V., "Bench and thermal vacuum testing of the JUICE-UVS microchannel plate detector system," Proc. SPIE 11118, UV, X-Ray, and Gamma-Ray Space Instrumentation for Astronomy XXI, 111180 Q (9 September 2019); https://doi.org/10.1117/12.2529649

[8] Davis, M.W., Greathouse, T.K., Gladstone, G.R., Retherford, K.D., Slater, D.C., Stern, S.A., Versteeg, M.H., "Improved ground calibration results from Southwest Research Institute Ultraviolet Radiometric Calibration facility (UV-RCF)," Proc. SPIE 9144, Space Telescopes and Instrumentation 2014: Ultraviolet to Gamma Ray, 914433 (2014); https://doi.org/10.1117/12.2057043

[9] Davis, M.W., Gladstone, G.R., Giles, R.S., Greathouse, T.K., Molyneux, P.M., Raut, U., Retherford, K.D., Baldor, S., Versteeg, M.H., Freeman, M., Persson, K., Persyn, S.C., "Ground calibration results of the JUICE ultraviolet spectrograph", Proc. SPIE 11444, Space telescopes and Instrumentation 2020: Ultraviolet to Gamma Ray, 1144404 (13 December 2020); https://doi.org/10.1117/12.2562986 